\title{
Long-range singlet proximity effect in ferromagnetic nanowires
}

\author{
F. Konschelle \\ Kavli Institute of Nanoscience, Delft University of Technology, P.O. Box 5046, 2600 GA Delft, The Netherlands
}

\author{
J. Cayssol and A. Buzdin* \\ CPMOH, Université de Bordeaux, UMR 5798, 33405 Talence, France
}

(Received 16 September 2010; published 11 November 2010)

\begin{abstract}
Recently a long-ranged superconductor/ferromagnet (S/F) proximity effect has been reported in Co crystalline nanowires [J. Wang, M. Singh, M. Tian, N. Kumar, B. Liu, C. Shi, J. K. Jain, N. Samarth, T. E. Mallouk, and M. H. W. Chan, Nat. Phys. 6, 389 (2010)]. Since the authors take care to avoid the existence of magnetic domains, the triplet character of the long-ranged proximity effect is improbable. Here we demonstrate that in the one-dimensional ballistic regime the standard singlet S/F proximity effect becomes long ranged. We provide an exact solution for the decay of the superconducting correlations near critical temperature $\left(T_{c}\right)$ and for arbitrary impurities concentration. In particular, we find a specific regime, between the diffusive and ballistic ones, where the decay length is simply the electronic mean-free path. Finally possible experiments which could permit to elucidate the nature of the observed long-ranged proximity effect in Co nanowires are discussed.
\end{abstract}

DOI: 10.1103/PhysRevB.82.180509

PACS number(s): 74.45.+c, 74.78.Fk, 85.25.Cp

It is well known that the superconducting proximity effect in a diffusive ferromagnetic $(\mathrm{F})$ metal is rather short ranged due to the large value of the ferromagnetic exchange field $E_{e x} \sim(500-5000) \mathrm{K}$ acting on the electron spins. In the ferromagnet, the singlet Cooper pair wave function experiences an oscillatory decay whose characteristic length $\xi_{f}=\sqrt{D / E_{e x}}$ enters the nanoscopic range for typical values of the diffusion constant $D$ and exchange field in the ferromagnet., This is in a sharp contrast with the corresponding decaying length in normal metals $\xi_{n}=\sqrt{D / T} \gg \xi_{f}$, which can reach microns at low-enough temperatures.

The presence of the nonuniform magnetization may strongly modify the superconductor/F $(\mathrm{S} / \mathrm{F})$ proximity effect for singlet superconductors and in the diffusive regime the induced triplet correlations can penetrate at the large distance $\xi_{n}{ }^{3}$ In the clean limit and in the presence of domains, the triplet proximity effect also increases the decay length up to the thermal decay length $\xi_{n b}=v_{F} / T$, ${ }^{4}$ where $v_{F}$ is the Fermi velocity. The long-ranged proximity effect has been observed in Ho (Ref. 5) and in the half metal $\mathrm{CrO}_{2} \cdot{ }^{6,7}$ Probably its origin is related with the presence of magnetic domains in Ho and with magnetic scattering at the S/F interface in the case of $\mathrm{CrO}_{2}$. Recently the controlled transition between the short ranged singlet and the long ranged triplet proximity effect has been reported in Co-based Josephson junctions using $\mathrm{Co} / \mathrm{Ru} / \mathrm{Co}$ (Ref. 8) and $\mathrm{Ho} / \mathrm{Co} / \mathrm{Ho}$ (Ref. 9) as magnetic barriers, respectively. Previously the $200 \mathrm{~nm}$ decay length for the coherence effect was observed in a system with mesoscopic Co wire. ${ }^{10}$ The geometry of the Co electrodes in the setup of Ref. 10 was nevertheless two-dimensional (2D)-like and probably the observed long ranged proximity effect was related with the presence of the magnetic domains which generated the triplet superconducting correlations. ${ }^{3}$

Very recently the Josephson current through the Co crystalline nanowires as long as $600 \mathrm{~nm}$ was reported. ${ }^{11}$ Interestingly the experiments on the Co crystalline nanowires ${ }^{11}$ were performed after applying a strong magnetic field which makes improbable the existence of the magnetic domains. The diameters of the Co nanowires ${ }^{11}$ were 40 and $80 \mathrm{~nm}$ and the proximity effect was substantially weaker for thicker nanowires. Note that in the S/F/S structures with Co film as a $\mathrm{F}$ layer, the long ranged proximity effect is absent and the characteristic decay length of superconductivity is around 5 $\mathrm{nm} .{ }^{12,13}$ Therefore the nanoscopic character of the Co wire seems to be crucial for the observation of the long-ranged proximity effect.

In this Rapid Communication we present a theoretical analysis of the $\mathrm{S} / \mathrm{F}$ proximity effect in the one-dimensional (1D) case taking in mind a thin Co nanowire. For this simple model it is possible to obtain an exact solution of Eilenberger equations with arbitrary impurity scattering. The decay of the superconducting correlations is governed by the electron mean-free path $l$. We believe that for the Co nanowire $l$ may be in the range (50-100) nm which could explain the longranged proximity effect observed in Ref. 11. We also discuss the alternative scenarios including dead layers or triplet correlations. Finally we suggest future experiments to discriminate between those different types of explanations.

The diffusive regime for the proximity effect in ferromagnets corresponds to the limit $E_{e x} \tau \ll 1,{ }^{14}$ which could be realized in weak ferromagnets with $\mathrm{CuNi}$ or $\mathrm{PdNi}$ alloys as $\mathrm{F}$ layer ${ }^{15,16}$ but not in the systems with strong ferromagnet such as $\mathrm{Co}, \mathrm{Ni}$, or Fe. ${ }^{12,13}$ However in ballistic regime the $\mathrm{S} / \mathrm{F}$ proximity effect is substantially different. The Cooper pair wave function oscillates inside the ferromagnet at the characteristic length $\xi_{f b}=v_{F} / E_{e x}$ (Ref. 17) but it decays much slowly: in three-dimensional system as $\sim\left(\xi_{f b} / x\right)$, where $x$ is the distance from the $\mathrm{S} / \mathrm{F}$ interface, in $2 \mathrm{D}$ systems as $\sim \sqrt{\left(\xi_{f b} / x\right)}$, and no decay in 1D case. ${ }^{18,19}$ Sure this statement is true for the distances $x$ smaller than thermal decay length $\xi_{n b}=v_{F} / T$ and mean-free path $l=v_{F} \tau$, where $\tau$ is the average time between impurity scattering events. Otherwise the range of the proximity effect will be fixed by the smallest length. ${ }^{20-22}$

Near the superconducting critical temperature, the Eilen- 
berger equations ${ }^{23}$ may be linearized and they read for the $1 \mathrm{D}$ case

$$
\left( \pm \frac{v_{F}}{2} \frac{\partial}{\partial x}+\omega+i E_{e x}+\frac{1}{2 \tau}\right) f_{ \pm}=\frac{1}{4 \tau}\left(f_{+}+f_{-}\right),
$$

where $f_{+}$and $f_{-}$are the anomalous Eilenberger Green's functions for $v_{F}>0$ and $v_{F}<0$, respectively, and $\omega$ is the fermionic Matsubara frequency. Note that $\left(f_{+}+f_{-}\right) / 2$ is simply the average over the Fermi surface in the 1D case.

After introducing the symmetric and antisymmetric combinations $F_{s}=\left(f_{+}+f_{-}\right) / 2$ and $F_{a}=\left(f_{+}-f_{-}\right) / 2$, one obtains that $F_{s}$ obeys the following second-order differential equation:

$$
\left(\omega+i E_{e x}\right) F_{s}-\frac{v_{F}^{2}}{4\left(\omega+i E_{e x}+\frac{1}{2 \tau}\right)} \frac{\partial^{2} F_{s}}{\partial x^{2}}=0
$$

while the antisymmetric combination can be obtained from

$$
F_{a}=-\frac{v_{F}}{2}\left(\omega+i E_{e x}+\frac{1}{2 \tau}\right)^{-1} \frac{\partial F_{s}}{\partial x} .
$$

Let us now sketch two limiting cases of Eq. (2). In the diffusive limit $E_{e x} \tau \ll 1$, Eq. (2) becomes

$$
\left(\omega+i E_{e x}\right) F_{s}-\frac{v_{F}^{2} \tau}{2} \frac{\partial^{2} F_{s}}{\partial x^{2}}=0,
$$

which is the Usadel equation with the diffusion coefficient $D=v_{F}^{2} \tau$. In the ballistic limit $E_{e x} \tau \gg 1$, Eq. (2) becomes

$$
\left(\omega+i E_{e x}\right)^{2} F_{s}-\frac{v_{F}^{2}}{4} \frac{\partial^{2} F_{s}}{\partial x^{2}}=0,
$$

which contains the same information as the Eilenberger equation Eq. (1) in the absence of disorder $(1 / \tau=0)$.

Solving the two previous Eqs. (4) and (5) for $E_{e x} \gg T_{c}$ shows that in the diffusive regime the characteristic oscillatory and damped behavior of $F_{s}$ occur with the same characteristic length $\xi_{f}$, whereas the ballistic regime may exhibit no attenuation at all. In the following, we investigate the effect of a small amount of impurities into the ferromagnetic wire. We show that this regime is characterized by two distinct lengths: the ballistic ferromagnetic oscillation one $\xi_{f b}$ and the damped one $l$ induced by the impurities into the wire.

The general solution of Eq. (2) for arbitrary impurity scattering is simply

$$
F_{s}=A e^{q x}+B e^{-q x}
$$

with

$$
q^{2}=\frac{4}{v_{F}^{2}}\left(\omega+i E_{e x}+\frac{1}{2 \tau}\right)\left(\omega+i E_{e x}\right) .
$$

Using Eq. (3) and the approximation $E_{e x} \tau \gg 1$, one obtains the antisymmetric anomalous propagator

$$
F_{a}=-A e^{q x}+B e^{-q x} .
$$

Assuming superconducting electrodes much thicker than the $\mathrm{F}$ nanowire, we apply the rigid boundary condition $f_{+}(x=-L / 2)=\Delta e^{-i \varphi / 2} / \omega$. Then we find the Eilenberger propagator for the superconducting correlations originating from the left electrode

$$
f_{+}=\frac{\Delta}{\omega} e^{-i \varphi / 2} e^{-q(x+L / 2)},
$$

where we suppose the temperature close to $T_{c}$. The similar condition $f_{-}(x=L / 2)=\Delta e^{-i \varphi / 2} / \omega$ yields the correlations from the right superconductor

$$
f_{-}=\frac{\Delta}{\omega} e^{i \varphi / 2} e^{q(x-L / 2)} .
$$

We now evaluate the Josephson supercurrent for this singlechannel situation

$$
\begin{aligned}
I & =\pi e \nu_{0} T \sum_{\omega} v_{F} I m\left(f_{-} f_{-}-f_{+} f_{+}\right) \\
& =2 \pi e \nu_{0} v_{F} T \sum_{\omega}\left(\frac{\Delta}{\omega}\right)^{2} \cos \left(\frac{2 E_{e x} L}{v_{F}}\right) e^{-L / 2 l} \sin \varphi \\
& =\frac{\pi}{2} e \nu_{0} v_{F} \frac{\Delta^{2}}{T_{c}} \cos \left(\frac{2 E_{e x} L}{v_{F}}\right) e^{-L / 2 l} \sin \varphi,
\end{aligned}
$$

where $\nu_{0}=k_{F} / \pi / E_{F}=\left(E_{F} a\right)^{-1}$ is the one-dimensional density of states per unit length (including two spin directions).

In the pure limit, $l \gg L$, the critical current exhibits undamped oscillations

$$
\begin{aligned}
I_{c 0} & =2 \pi e \nu_{0} v_{F} T \sum_{\omega}\left(\frac{\Delta}{\omega}\right)^{2} \cos \left(\frac{2 E_{e x} L}{v_{F}}\right) \\
& =\frac{\pi}{2} e \nu_{0} v_{F} \frac{\Delta^{2}}{T_{c}} \cos \left(\frac{2 E_{e x} L}{v_{F}}\right)
\end{aligned}
$$

whose amplitude gives an estimate for the single-channel critical current. The above result was derived near the critical temperature. Concerning order of magnitudes, it can be safely extrapolated to low temperatures by substituting $\Delta=\Delta(T)$ by $\Delta(T=0) \simeq T_{c}$. We introduce the quantum of resistance $R_{N}=h / e^{2}$ and find

$$
I_{c 0} \sim \frac{\pi}{2} e \nu_{0} v_{F} \frac{\Delta^{2}}{T_{c}} \sim \frac{T_{c}}{e R_{N}} .
$$

Hence we can estimate for the experimental conditions ${ }^{11}$

$$
e R_{N} I_{c 0} \sim 1 \mathrm{mV} \text { and so } I_{c 0} \sim 40 \mathrm{nA} \text {. }
$$

This maximal current per mode is damped by the exponential factor $e^{-L / 2 l}$.

For a metallic nanowire with a cross section $S$, the number $M$ of transverse channels typically amounts $S / a^{2} \simeq(d / a)^{2} \simeq\left(10^{4}-10^{5}\right)$. This relatively large number of channels may compensate the exponential suppression of the critical current which can be evaluated as $I_{c}=M e^{-L / 2 l} I_{c 0}$. In experiment, ${ }^{11}$ the critical current exceeds $10 \mu \mathrm{A}$ and $M \simeq 10^{5} \quad[d=(40-80) \mathrm{nm}, \quad L=600 \mathrm{~nm}$, and $a=0.1 \mathrm{~nm}]$ thereby requiring a rather long mean-free path, namely, $l \geq 60 \mathrm{~nm}$. On the basis of the Drude model, ${ }^{11}$ the estimate of the mean-free path $l$ is quite sample dependent with values ranging in the window $l \sim(2-10) \mathrm{nm}$. In our approach such short mean-free paths would lead to a very strong suppres- 
sion of the current. Nevertheless we might question if those values $l \sim(2-10) \mathrm{nm}$ represent the genuine mean-free path inside the ferromagnet. Indeed the authors ${ }^{11}$ note an important diffusion of the $\mathrm{W}$ atoms inside Co nanowires. Therefore the region near the W/Co interface may contribute to the measured resistivity and the genuine or intrinsic mean-free path $l$ (defined between the contact regions) of the Co nanowire may be substantially higher than the simple Drude estimate.

Long ranged triplet proximity effect, caused by some magnetic inhomogeneity, is also a possible scenario to explain the experiment. ${ }^{11}$ A different kind of Josephson junctions $\mathrm{S} / \mathrm{X}_{1} / \mathrm{F} / \mathrm{X}_{2} / \mathrm{S}$ with three magnetic layers $\left(\mathrm{X}_{1}, \mathrm{~F}, \mathrm{X}_{2}\right)$ has been proposed ${ }^{4}$ and successfully implemented in a controlled way. ${ }^{8,9}$ A central region $\mathrm{F}$ is used to suppress singlet Josephson current while the side domains create triplet correlations. In the original theoretical setup, the $\mathrm{X}_{1}, \mathrm{~F}$, and $\mathrm{X}_{2}$ were three monodomains with noncollinear magnetizations. In the recent experiment, ${ }^{8} \mathrm{X}_{1}$ and $\mathrm{X}_{2}$ were layers of weak ferromagnets while $\mathrm{F}$ was itself a $\mathrm{Co}$ bilayer, $\mathrm{Co} / \mathrm{Ru} / \mathrm{Co}$. In Ref. 9 the synthetic $\mathrm{Ho} / \mathrm{Co} / \mathrm{Ho}$ ferromagnetic trilayer was used to the same purpose. More specifically it was predicted ${ }^{4}$ and confirmed experimentally ${ }^{8}$ that the production of triplet correlations is maximal for an optimal size of the $X_{1}$ and $X_{2}$ layers, which is on the order of $\xi_{f b}$. The authors in Ref. 11 mention that though the Co nanowires must be single domain, the inhomogeneous magnetic moments may be produced in the W-Co contact regions. Following Refs. 4, 8, and 9 the size of such regions must be of order $\xi_{f}$ to maximize the triplet long-ranged effect. Nevertheless this contact region is likely to be of the atomic size $a$ and in such a case the triplet Josephson current would be reduced by a factor $\left(a / \xi_{f b}\right)^{2} \simeq\left(10^{-2}-10^{-1}\right)$. The optimal triplet supercurrent is $M \simeq 10^{5}$ times the single-channel $\mathrm{S} / \mathrm{N} / \mathrm{S}$ supercurrent $(0.04 \mu \mathrm{A})$. Hence our estimation of the triplet effect in (Ref. 11) is $I_{c}=(4-40) \mu \mathrm{A}$, which is still in agreement with the reported supercurrents.

In order to discriminate between the two scenarios described above, it is crucial to know whether the Co nanowires are in the diffusive or in the ballistic limit, and also to have an experimental determination of the Josephson current-phase relation. ${ }^{1,2}$ Indeed the presence or absence of a second harmonic at low temperature allows a distinction be- tween those two scenarios. In the singlet correlation scenario, the second harmonic will be strongly suppressed by disorder (by an additional factor $e^{-L / 2 l}$ relative to the first harmonic) while for triplet proximity effect the second harmonic is expected to be of the same order of magnitude as the first one, at low temperature.

Besides, it would also be interesting to know if the $S / F / S$ structures with Co nanowire may be in the $\pi$ state. This can be determined by realizing a superconducting quantum interference device with the Co nanowire Josephson junction as one arm and a standard Josephson junction on the other arm.

Note that a third possible scenario might involve a dead layer at the surface of the Co nanowire, where magnetism is strongly suppressed thereby leading to the proximity effect reported in Ref. 11. Indeed the Co wires are surrounded by an insulating oxide coating and a dead layer (without ferromagnetic order) might appear between this insulating layer and the Co wire core. The presence of such a dead layer was already signaled in Co films with typical size $1 \mathrm{~nm} .{ }^{12}$ In the extreme case, the magnetism is completely absent within this layer and the corresponding supercurrent would not have the exponential decay. In such a case the number of channels would be $M_{\text {surf }}=d / a \simeq 10^{3}$ and the critical current would reach quite large values $10^{2} \mu \mathrm{A}$, even for long nanowires. Note that in presence of such a dead layer and at low temperature, the second harmonic of the current-phase relation would be as large as the first harmonic, similar to the case of the triplet long-ranged proximity effect.

In conclusion, we have found that the long-ranged S/F proximity effect reported recently in Ref. 11 can be explained by three distinct scenarios. In the first scenario, the Josephson current is associated with weakly damped singlet superconducting correlations. In the second scenario, the contact regions produce triplet correlations thereby leading to long ranged proximity effect. Finally in a third scenario a nonmagnetic dead layer provides a channel for long-ranged propagation of the superconducting correlations. We further suggest to realize the low-temperature determination of the current-phase relation to discriminate between those scenarios.

This work was supported by the Agence Nationale de la Recherche under Grant No. ANR-07-NANO-011-05: ELECEPR.

\footnotetext{
*Also at Institut Universitaire de France.

${ }^{1}$ A. I. Buzdin, Rev. Mod. Phys. 77, 935 (2005).

${ }^{2}$ A. A. Golubov, M. Yu. Kupriyanov, and E. Il'ichev, Rev. Mod. Phys. 76, 411 (2004).

${ }^{3}$ F. S. Bergeret, A. F. Volkov, and K. B. Efetov, Rev. Mod. Phys. 77, 1321 (2005).

${ }^{4}$ M. Houzet and A. I. Buzdin, Phys. Rev. B 76, 060504(R) (2007).

${ }^{5}$ I. Sosnin, H. Cho, V. T. Petrashov, and A. F. Volkov, Phys. Rev. Lett. 96, 157002 (2006).

${ }^{6}$ R. S. Keizer, S. T. B. Goennenwein, T. M. Klapwijk, G. Miao, G.
}

Xiao, and A. Gupta, Nature (London) 439, 825 (2006).

${ }^{7}$ M. S. Anwar, F. Czeschka, M. Hesselberth, M. Porcu, and J. Aarts, Phys. Rev. B 82, 100501 (2010).

${ }^{8}$ T. S. Khaire, M. A. Khasawneh, W. P. Pratt, Jr., and N. O. Birge, Phys. Rev. Lett. 104, 137002 (2010).

${ }^{9}$ J. W. A. Robinson, J. D. S. Witt, and M. G. Blamire, Science 329, 59 (2010).

${ }^{10}$ M. Giroud, H. Courtois, K. Hasselbach, D. Mailly, and B. Pannetier, Phys. Rev. B 58, R11872 (1998).

${ }^{11}$ J. Wang, M. Singh, M. Tian, N. Kumar, B. Liu, C. Shi, J. K. Jain, N. Samarth, T. E. Mallouk, and M. H. W. Chan, Nat. Phys. 6, 
389 (2010).

${ }^{12}$ J. W. A. Robinson, S. Piano, G. Burnell, C. Bell, and M. G. Blamire, Phys. Rev. Lett. 97, 177003 (2006).

${ }^{13}$ M. A. Khasawneh, W. P. Pratt, Jr., and N. O. Birge, Phys. Rev. B 80, 020506 (2009).

${ }^{14}$ A. Buzdin and I. Baladie, Phys. Rev. B 67, 184519 (2003).

${ }^{15}$ V. V. Ryazanov, V. A. Oboznov, A. Yu. Rusanov, A. V. Veretennikov, A. A. Golubov, and J. Aarts, Phys. Rev. Lett. 86, 2427 (2001).

${ }^{16}$ T. Kontos, M. Aprili, J. Lesueur, F. Genêt, B. Stephanidis, and R. Boursier, Phys. Rev. Lett. 89, 137007 (2002).

${ }^{17}$ A. I. Buzdin, L. N. Bulaevskii, and S. V. Panyukov, Pis'ma Zh.
Eksp. Teor. Fiz. 35, 147 (1982) [JETP Lett. 35, 178 (1982)].

${ }^{18}$ F. Konschelle, J. Cayssol, and A. I. Buzdin, Phys. Rev. B 78, 134505 (2008).

${ }^{19}$ J. Cayssol and G. Montambaux, Phys. Rev. B 70, 224520 (2004).

${ }^{20}$ L. N. Bulaevskii, A. I. Buzdin, and S. V. Panyukov, Solid State Commun. 44, 539 (1982).

${ }^{21}$ F. S. Bergeret, A. F. Volkov, and K. B. Efetov, Phys. Rev. B 64, 134506 (2001).

${ }^{22}$ J. Linder, M. Zareyan, and A. Sudbø, Phys. Rev. B 79, 064514 (2009).

${ }^{23}$ G. Eilenberger, Z. Phys. 214, 195 (1968). 